minority communities in Florida to gauge the awareness and understanding of COVID-19, and the barriers and facilitators for participation in COVID-19 research studies. These communities include but are not limited to Latinx and Black populations in South and Central Florida, and Black communities in North Florida. The outcomes will help shape strategies for outreach and dissemination activities and minority recruitment plans to promote participation of minorities into vaccine and therapeutic trials. RESULTS/ ANTICIPATED RESULTS: An estimated 75-125 participants will be recruited for focus groups. Four focus groups with minority communities have been conducted and the results are being analyzed. A common Community-Based Needs Assessment survey is being finalized and will be deployed across the 11 states that are part of the national CEAL consortium. Community Health Workers are being engaged to support outreach and dissemination to educate targeted communities on COVID-19 research and the importance of participation in COVID trials. To date, 243 CHWs and 880 community members have been engaged. Minority participation in COVID-19 vaccine trials at University of Miami has been higher than the national average. DISCUSSION/SIGNIFICANCE OF FINDINGS: The FL-CEAL Alliance has successfully demonstrated a coordinated effort to engage minority communities affected by COVID. Through strategic geographic partnerships, FL-CEAL will positively impact minority communities throughout the state that has one of the most diverse populations in the nation.

50565

CTSA collaboration to support K-12 school re-opening in the COVID-19 pandemic

Moira Inkelas ${ }^{1}$, Onyebuchi A. Arah ${ }^{1}$, Vladimir G. Manuel ${ }^{2}$, Roch Nianogo ${ }^{1}$, "Douglas E. Morrison ${ }^{1}$, Nathaniel Anderson ${ }^{1}$, Defne Yilmaz ${ }^{1}$ and Tony Kuo ${ }^{1,2}$

${ }^{1}$ Fielding School of Public Health, University of California Los Angeles and ${ }^{2}$ David Geffen School of Medicine, University of California Los Angeles

ABSTRACT IMPACT: The mobilization of a CTSA-sponsored team with multi-disciplinary translational science expertise enabled the university to provide a range of T1-T4 expertise to a large, complex school district that resulted in permanent learning and data science infrastructure. OBJECTIVES/GOALS: The Clinical Translational Science Institute (CTSI) formed a multidisciplinary science team to provide expertise in support of the re-opening of in-person learning in the second-largest U.S. school district during the COVID-19 pandemic. METHODS/STUDY POPULATION: The assembled interdisciplinary science team provided expertise in epidemiology, machine learning, causal inference and agent-based modeling, data and improvement science, biostatistics, clinical and laboratory medicine, health education, community engagement, and experience in outbreak investigation and management. The team included TL1 pre and postdoctoral fellows and mobilized scientists from multiple professional schools and T1-T4 stages of translational research. RESULTS/ANTICIPATED RESULTS: Tangible outcomes achieved using this team approach included the development of practical metrics for use in the school community, a learning process, the integration of preventive design elements into a testing and tracing program, and targeted and data-driven health education. The team, for example, generated new data displays for community engagement and collaborated with the school district in their use to visualize, learn from, and act on variation across a 700 square mile region. DISCUSSION/SIGNIFICANCE OF FINDINGS: Novel translational methods can be used to establish a learning environment and data science infrastructure that complements efforts of public health agencies to aid schools in the COVID-19 pandemic. These new capabilities apply to COVID-19 testing and vaccines and can be mobilized for future population health challenges faced by school districts.

79602

\section{Designing and Implementing an Assessment of Collaboration for a Clinical and Translational Research Community Advisory Board}

Paul Estabrooks, Keyonna King, Dave Palm and Heidi Keeler University of Nebraska Medical Center

ABSTRACT IMPACT: This abstract presents a generalizable process to evaluate, and act on, community advisory board perceptions of collaboration effectiveness to improve clinical and translational research network function. OBJECTIVES/GOALS: Community advisory boards (CAB) play an important role in facilitating relevant and externally valid, clinical and translational research (CTR). The objective of this presentation is to describe a participatory process to derive collaboration metrics that can be used to assess CAB effectiveness. METHODS/STUDY POPULATION: During the 4 th and 5 th years of the Great Plains IDeA CTR award, we used a mixed-methods approach that included CAB (1) discussions related to the need to assess collaboration effectiveness, (2) review of the validated Wilder Collaboration Inventory to identify factors that, if maximized, would improve a sense of team science and enhance productivity, (3) planning for assessment frequency and follow-up processes, and (4) review of collaboration data to determine necessary actions for improvement. Qualitative data were gathered across components of the mixed-methods approach. Quantitative data were collected and reviewed by the $\mathrm{CAB}(\mathrm{n}=11$ members) during the 1 st quarter of award year 5. RESULTS/ANTICIPATED RESULTS: CAB members expressed an interest in assessing collaboration effectiveness, identified important factors to assess, and agreed that annual assessment and follow-up would be appropriate. Key factors identified and assessed (5-point agreement scale-higher score reflects stronger agreement) were 1) mutual respect/trust $(\mathrm{m}=4.2) ; 2)$ appropriate cross section of members $(\mathrm{m}=3.6) ; 3)$ a shared stake in the process and outcomes $(\mathrm{m}=3.9) ; 4)$ flexibility in decision-making/collaboration $(\mathrm{m}=4.1) ; 5)$ roles and policy guidelines $(\mathrm{m}=3.6) ; 6)$ open communication $(\mathrm{m}=3.9)$; 7) goal achievement $(\mathrm{m}=4.0)$; 8$)$ shared vision $(\mathrm{m}=4.0)$; and 9) skilled leadership $(m=4.4)$. DISCUSSION/SIGNIFICANCE OF FINDINGS: CAB reflection on the initial collaboration assessment resulted in developing plans to broaden membership and clarify roles and policy guidelines related to $\mathrm{CAB}$ participation. There was strong consensus related to the utility of this assessment approach.

\section{3}

Network Evaluation of a Community-Campus Partnership: Applying a Systems Science Lens to Evaluating Collaboration and Translation

John D. Prochaska ${ }^{1}$, Sharon Croisant ${ }^{1}$, Lesley C. Sommer ${ }^{2}$, Neil Treble $^{2}$, Krista Bohn ${ }^{1}$, Lori Wiseman ${ }^{1}$, Chantele Singleton ${ }^{1}$ and Lance Hallberg ${ }^{1}$

${ }^{1}$ University of Texas Medical Branch and ${ }^{2} \mathrm{REACH}$ Coalition of Galveston County

ABSTRACT IMPACT: Using network analysis and a systems science lens, UTMB's Institute for Translational Sciences is able to quantify the evolution of REACH (its Community-Campus Partnership) as 